Алгебра и анализ

Том. 17 (2005), вып. 2
St. Petersburg Math. J. Vol. 17 (2006), No. 2, Pages 315-323 S 1061-0022(06)00906-X

Article electronically published on February 20, 2006

\title{
ON APPROXIMATION OF A THREE-DIMENSIONAL CONVEX BODY BY CYLINDERS
}

\author{
V. V. MAKEEV
}

\begin{abstract}
New results on approximation of a convex body $K \subset \mathbb{R}^{3}$ by affine images of circular cylinders, parallelepipeds, hexagonal and octagonal regular (and some other) prisms are obtained.

Two of the theorems obtained are as follows $(V(K)$ denotes the volume of a body $\left.K \subset \mathbb{R}^{3}\right)$.

Theorem 1. Let $K$ be an arbitrary convex body in $\mathbb{R}^{3}$. There exists a regular octagonal prism an affine image of which is circumscribed about $K$ and has volume at most $3 \sqrt{2} V(K)$, and there exists a circular cylinder an affine image of which is circumscribed about $K$ and has volume at most $\frac{3 \pi}{2} V(K)$. For a tetrahedron $K$ both estimates are the best possible.

Theorem 2. Let $K$ be a centrally symmetric convex body in $\mathbb{R}^{3}$. There exists a regular octagonal prism, an affine image of which lies in $K$ and has volume at least $\frac{4}{9}(2 \sqrt{2}-2) V(K)$.
\end{abstract}

In what follows, by a convex body $K \subset \mathbb{R}^{n}$ (a figure $K \subset \mathbb{R}^{2}$ ) we mean a compact convex set with nonempty interior; by $S(K)$ and $V(K)$ we mean the area of $K \subset \mathbb{R}^{2}$ and the volume of $K \subset \mathbb{R}^{n}$, respectively. By $G_{k}\left(\mathbb{R}^{n}\right)$ (respectively, $G_{k}^{+}\left(\mathbb{R}^{n}\right)$ ), we mean the Grassmann manifold of $k$-planes (respectively, of oriented $k$-planes) in $\mathbb{R}^{n}$ passing through $0 \in \mathbb{R}^{n}$. Let $\gamma_{k}^{n}: E_{k}\left(\mathbb{R}^{n}\right) \rightarrow G_{k}\left(\mathbb{R}^{n}\right)$ (respectively, $\left(\gamma_{k}^{n}\right)^{+}: E_{k}^{+}\left(\mathbb{R}^{n}\right) \rightarrow G_{k}^{+}\left(\mathbb{R}^{n}\right)$ ) be the tautological bundle over the Grassmann manifold, in which the fiber over a plane is the same plane regarded as a $k$-dimensional subspace of $\mathbb{R}^{n}$. As usual, let $V_{k}\left(\mathbb{R}^{n}\right)$ be the Stiefel manifold of orthonormal $k$-frames in $\mathbb{R}^{n}$.

We say that a polyhedron $M$ is inscribed in a convex body $K \subset \mathbb{R}^{n}$ if the vertices of $M$ belong to the boundary of $K$. A polyhedron $M$ is circumscribed about $K$ if $K \subset M$ and $K$ intersects all faces of $M$.

\section{$\S 1$. A Lemma on Approximation of A CONVEX BODY By A CYLinder}

In the sequel, we need the following simple (and, apparently, well-known) statement.

Lemma 1. For every convex body $K \subset \mathbb{R}^{n}$ and a line $l$ in $\mathbb{R}^{n}$, there exists a convex cylinder $C$ of volume $V(C) \leq n V(K)$ that contains $K$, has ruling parallel to $l$, and is such that the line connecting two points where $K$ meets the bases of $C$ is parallel to $l$.

Proof. Consider the union $C^{\prime}$ of lines that are parallel to $l$ and intersect $K$. By construction, $C^{\prime}$ is an infinite convex cylinder. Let $A B$ be the longest chord $A B$ of $K$ parallel to $l$.

2000 Mathematics Subject Classification. Primary 52B10.

Key words and phrases. Volume, cylinder, prism, parallepiped. 


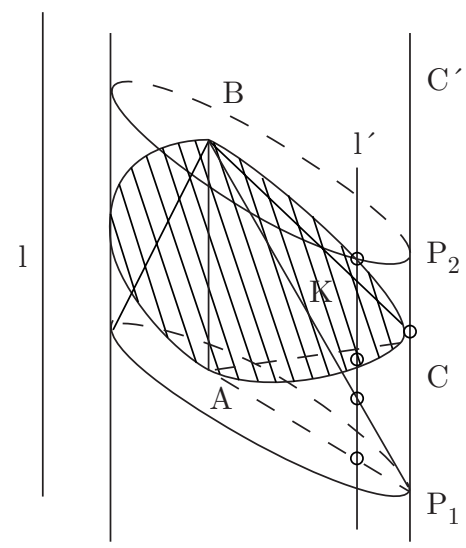

FIGURE 1.

Obviously, there exist two parallel support hyperplanes $P_{1}$ and $P_{2}$ for the body $K$ at $A$ and $B$. We show that $P_{1}$ and $P_{2}$ cut from $C^{\prime}$ a required cylinder $C$ containing $K$ (Figure 1).

Consider the cone $K_{1}$ with vertex $B$ and base coinciding with the base of $C$ that passes through the vertex $A$. Clearly, $V\left(K_{1}\right)=\frac{V(C)}{n}$.

We prove that $V(K) \geq V\left(K_{1}\right)$. It is obvious that the length of the intersection of $K$ with an arbitrary line $l^{\prime}$ parallel to $l$ does not exceed the length of the intersection of $l^{\prime}$ with the cone $K_{1}$ (Figure 1). Consequently, by the Cavalieri principle, we have $V(K) \geq V\left(K_{1}\right)$, which completes the proof of the lemma.

\section{§2. Approximation of a three-dimensional Convex body BY AFFINE-REGULAR HEXAGONAL PRISMS}

Theorem 1. 1. About an arbitrary convex body $K \subset \mathbb{R}^{3}$, an affine image of a regular hexagonal prism with a prescribed direction of a lateral edge can be circumscribed. The segment connecting the points at which the bases of the cylinder are tangent to $K$ is parallel to a lateral edge of the prism. The volume of this prism does not exceed $6 \mathrm{~V}(\mathrm{~K})$.

2. About an arbitrary convex body $K \subset \mathbb{R}^{3}$, an affine image of a regular hexagonal prism tangent to $K$ at the centers of the bases can be circumscribed.

Proof. 1. We note that the orthogonal projection of $K \subset \mathbb{R}^{3}$ onto a plane $P$ orthogonal to a given line $l \subset \mathbb{R}^{3}$ is inscribed into an affine-regular hexagon $S$.

Let $C$ be an infinite cylinder bounded by the six planes that pass through the sides of $S$ and are parallel to $l$. Let $A B$ be the longest chord of $K$ parallel to $l$. Then there are parallel support planes to $K$ passing through $A$ and $B$ and such that, taken together with the cylinder $C$, they bound the required prism.

The estimate for the volume of this prism is obtained by application of the lemma and the fact that the area of an affine-regular hexagon $S$ circumscribed about a plane convex figure does not exceed the doubled area of the latter.

2. It suffices to prove the second statement of the theorem for smooth strictly convex bodies. In the other cases, the theorem is obtained by passing to a limit.

In each plane $P$ through $O \in \mathbb{R}^{3}$, we mark its intersection point with the line containing a unique (by the strict convexity of $K$ ) longest chord of $K$ perpendicular to $P$. We have constructed a section of the tautological bundle $\gamma_{2}^{3}: E_{2}\left(\mathbb{R}^{n}\right) \rightarrow G_{2}\left(\mathbb{R}^{3}\right)$. Let $C_{1}$ be its support. 
In each plane $P$ in question, we mark also the centers of the affine-regular hexagons circumscribed about the orthogonal projection of the body $K$ onto the plane $P$. Let $C_{2}$ be the set of centers of all such hexagons in $E_{2}\left(\mathbb{R}^{3}\right)$. For a typical smooth body $K$, the set $C_{2}$ is a compact two-dimensional manifold that intersects a typical plane $P \subset E_{2}\left(\mathbb{R}^{3}\right)$ at an odd number of points, because (see [1]) an odd number of affine-regular hexagons can be circumscribed about a smooth convex figure in general position in the plane.

Thus, for a typical smooth strictly convex body $K$, the cycles $C_{1}$ and $C_{2}$ represent generators of $H_{2}\left(E_{2}\left(\mathbb{R}^{3}\right) ; \mathbb{Z}_{2}\right)$ and have intersection index 1 modulo 2. Therefore, $C_{1} \cap$ $C_{2} \neq \varnothing$, which proves the theorem.

Remarks. 1. The volume estimate in item 1 of the theorem is the best possible for a tetrahedron $K$ and an arbitrary direction of a lateral edge of the circumscribed hexagonal prism parallel to a line passing through the vertex of $K$ and a point of the face opposite to this vertex.

2. It seems plausible that a regular hexagonal prism can be circumscribed about an arbitrary convex body $K \subset \mathbb{R}^{3}$; however, the author was not able to prove this.

In [3], it was proved that a regular pentagon can be circumscribed about the orthogonal projection of a convex body $K \subset \mathbb{R}^{3}$ onto a plane; therefore, a regular pentagonal prism can be circumscribed about a convex body $K \subset \mathbb{R}^{3}$.

3. It can easily be seen that the volume of a tetrahedron lying in a regular hexagonal prism $C$ does not exceed $\frac{2}{9} V(C)$ and equality is attained if the skew edges of the tetrahedron are great diagonals of the bases. Is it true that each convex body $K \subset \mathbb{R}^{3}$ lies in an affine-regular hexagonal prism of volume at most $\frac{9}{2} V(K)$ ? The results of the next section imply that this is true if the constant $\frac{9}{2}$ is replaced by $\frac{45 \sqrt{2}+72}{28}<4.845$.

\section{§3. Approximation of a three-Dimensional Body BY AFFINE-REGULAR OCTAGONAL PRISMS}

Theorem 2. For an arbitrary convex body $K$ in $\mathbb{R}^{3}$, there exists a regular octagonal prism, an affine image of which is circumscribed about $K$ and has volume at most $3 \sqrt{2} V(K)$. For a tetrahedron $K$, the above estimate is the best possible.

Proof. In [1], it was proved that the projection of $K$ onto a plane along a line $l$ is inscribed into an affine-regular octagon.

By the lemma proved above, the body $K$ lies in a cylinder $C$ with ruling parallel to $l$ and $V(C) \leq 3 V(K)$. Then an affine-regular octagonal prism $C_{1}$ with base lying in the same plane and with the same direction of ruling is circumscribed about $C$. The base $T$ of the cylinder $C$ is inscribed into an affine-regular octagon $T_{1}$, which is the base of the prism $C_{1}$.

We prove that $S(T) \leq S\left(T_{1}\right) / \sqrt{2}$. By simple variational considerations, the ratio $S(T) / S\left(T_{1}\right)$ is minimal if $T$ is a polygon with vertices at the vertices of $T_{1}$, and, therefore, if $T$ is a parallelogram inscribed in $T_{1}$, and $S(T) / S\left(T_{1}\right)=1 / \sqrt{2}$.

To complete the proof, it suffices to observe that a tetrahedron of maximal volume inscribed in a regular octagonal prism has perpendicular diameters of the bases as pairs of its skew edges, and its volume is equal to $\frac{1}{3 \sqrt{2}}$ of the volume of the prism.

Corollary 1. Each convex body $K \subset \mathbb{R}^{3}$ lies in a parallelepiped of volume not exceeding $3\left(1+\frac{\sqrt{2}}{2} V(K)\right.$.

Indeed, a prism that exists by the above theorem can be inscribed in the parallelepiped that has a volume indicated (Figure 2) and is bounded by the planes passing through the bases of the prism and the planes passing through every other lateral face. 


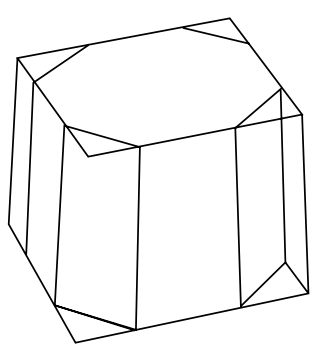

FIGURE 2.

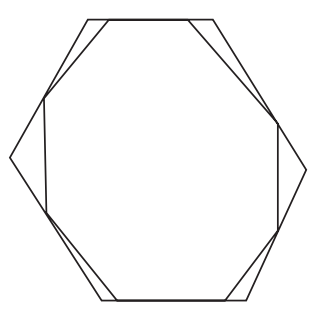

FiguRE 3.

1. We note that the problem of approximation of a convex body $K \subset \mathbb{R}^{3}$ by a parallelepiped from inside was solved in [2], where it was proved that every convex body $K \subset \mathbb{R}^{3}$ contains a parallelepiped of volume not less than $\frac{2}{9} V(K)$ and that this estimate cannot be improved if $K$ is a tetrahedron.

2. About any convex body $K \subset \mathbb{R}^{3}$, an affine-regular hexagonal prism of volume at most $\frac{45 \sqrt{2}+72}{28} V(K)<4.845 V(K)$ can be circumscribed.

To prove this statement, we consider an affine-regular octagonal prism $C$ of volume not exceeding $3 \sqrt{2} V(K)$ and containing $K$, and circumscribe about it an affine-regular hexagonal prism $C_{1}$ with the same bases and the same direction of the lateral edges. The bases of this prism are affine-regular hexagons circumscribed about the octagonal bases of $C$ as shown in Figure 3. Simple calculations show that $V\left(C_{1}\right)=\frac{15+12 \sqrt{2}}{28} V(C)$, which implies the required estimate.

3. About a convex body $K \subset \mathbb{R}^{3}$, a hexagonal prism with centrally symmetric base and volume at most $\frac{6+9 \sqrt{2}}{4} V(K)<4.6822 V(K)$ can be circumscribed.

Such a prism is obtained by deleting two symmetric lateral faces from the affine-regular octagonal prism constructed in the above theorem.

\section{§4. Approximation of a three-Dimensional COnVEX body BY AN AFFINE IMAGE OF A CIRCULAR CYLINDER}

In [5], it was proved that, for every convex body $K$ in $\mathbb{R}^{3}$ there exists a circular cylinder an affine image of which is contained in $K$ and has volume at least $\frac{4 \pi}{27 \sqrt{3}} V(K)$, and that this estimate cannot be improved if $K$ is a tetrahedron.

This theorem solves the problem of approximation from inside of a convex body $K \subset$ $\mathbb{R}^{3}$ by an affine image of a circular cylinder. The following theorem solves the same question for approximations of a convex body $K \subset \mathbb{R}^{3}$ from outside. 


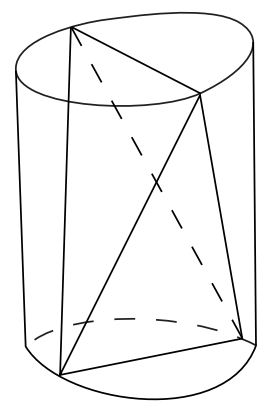

FiguRE 4.

Theorem 3. For every convex body $K$ in $\mathbb{R}^{3}$, there exists a circular cylinder an affine image of which contains $K$ and has volume at most $\frac{3 \pi}{2} V(K)$. For a tetrahedron $K$, this estimate cannot be improved.

To prove the first statement, it suffices to circumscribe an elliptic cylinder about an affine-regular octagonal prism existing by Theorem 2 . The second statement follows from the fact that, for a tetrahedron of maximal volume inscribed in a circular cylinder, two perpendicular diameters of the base of the cylinder are skew edges of the tetrahedron (Figure 4), and the volume of this tetrahedron is $\frac{2}{3 \pi}$ of the volume of the cylinder.

Remark. Among all cylinders, the elliptic ones are the worst approximable by inscribed tetrahedrons.

Proposition. For every convex cylinder $C$ in $\mathbb{R}^{3}$, there exists an inscribed tetrahedron of volume at least $\frac{2}{3 \pi} V(C)$; equality occurs only for elliptic cylinders.

Let $C_{1}$ be the lower base of a convex cylinder $C \subset \mathbb{R}^{3}$. By [6, p. 69], a quadrangle $A B C D$ of area at least $\frac{2}{\pi} S(C)$ is inscribed in $C_{1}$, and the inequality can be strict only if $C_{1}$ is not an ellipse. Let $A_{1} B_{1} C_{1} D_{1}$ be the translate of $A B C D$ inscribed in the upper base of $C$. Then $A C B_{1} D_{1}$ is a tetrahedron of volume at least $\frac{2}{3 \pi} V(C)$ inscribed in $C$.

\section{§5. Approximation of a Centrally symmetric three-dimensional CONVEX BODY BY AFFINE REGULAR OCTAGONAL PRISMS}

In what follows, we use the abbreviation c.s.c.b. $K$ for "centrally symmetric convex body $K "$.

Proposition. Every c.s.c.b. $K \subset \mathbb{R}^{3}$ is contained in a circumscribed affine image of a regular octagonal prism tangent to $K$ at the centers of the bases and having a lateral edge with a prescribed direction.

The proof is similar to that of Theorem 2 (see $\S 3$ ) and follows from the fact 7 that about every centrally symmetric convex figure in a plane one can circumscribe an affine image of a regular octagon, and the longest chord of a c.s.c.b $K \subset \mathbb{R}^{3}$ can be drawn through its center in an arbitrary direction.

Remarks. 1. Choosing the direction of a lateral edge of the prism coinciding with the direction of a diameter of $K$, we can construct a direct prism of the type indicated in the theorem.

2. As in the theorem, the volume of the circumscribed prism is less than or equal to $3 \sqrt{2} V(K)$. Moreover, if $K$ is an octahedron and a lateral edge has the same direction as a diagonal of $K$, then the above estimate is the best possible. 
Theorem 3. Every c.s.c.b. $K \subset \mathbb{R}^{3}$ contains an inscribed affine image of a regular octagonal prism with the same center of symmetry and with volume at least $\frac{4(2 \sqrt{2}-2)}{9} V(K)>$ $0.3681 V(K)$.

We need the following statement.

Lemma 2. If a convex figure $K \subset \mathbb{R}^{2}$ is circumscribed about a regular octagon $S$, the distance between opposite sides of which is 1 , then $S(K) \leq 1$.

Drawing the support lines to $K$ at the vertices of the octagon $S$, we may assume that $K$ is an octagon circumscribed about $S$.

By standard variational considerations, we see that either the sides of a circumscribed octagon $K$ of maximal area are split by the vertices of $S$ into two equal parts, or some two sides of $K$ contain sides of $S$.

Any octagon $K$ with the sides' midpoints at the vertices of $S$ has vertices obtained from a certain point of the plane by successive reflections with respect to the vertices of $S$. (One of the vertices of $K$ can lie on a side of $S$.) It can easily be proved that the areas of all octagons $K$ in question circumscribed about $S$ are equal and do not exceed 1.

A simple but time-consuming consideration of the cases where two or three sides of $S$ belong to the sides of $K$ shows that in these cases we also have $S(K) \leq 1$.

Now we proceed to the proof of the theorem.

We show that, for each plane $P \in G_{2}\left(\mathbb{R}^{3}\right)$, we can find a pair of planes $P_{1}$ and $P_{2}$ that depend continuously on $P$, are symmetric relative to the center of symmetry of $K$, and satisfy the inequality $S\left(P_{1} \cap K\right) \cdot d \geq \frac{4}{9} V(K)$, where $d$ is the distance between $P_{1}$ and $P_{2}$.

We prove this for a smooth strictly convex body $K$; in the other cases the proof is obtained by passage to a limit.

We choose $P \in G_{2}\left(\mathbb{R}^{3}\right)$ and consider the Swartz rounding of $K$ with respect to a line perpendicular to $P$. We obtain a smooth strictly convex body of revolution $K_{1}$ that is reflection symmetric with respect to a plane $P_{0}$ parallel to $P$. Taking the support planes to $K_{1}$ at all boundary points of $K_{1}$ at a distance $x>0$ from the plane $P_{0}$, we obtain bicones of revolution symmetric with respect to $P_{0}$ (Figure 5). By simple monotonicity and continuity arguments, there exists a single number $x(P)>0$ depending on $P$ such that $h(P)=6 x(P)$, where $h(P)$ is the height of the bicone $C$ constructed by $x(P)$. Let $P_{1}$ and $P_{2}$ be planes parallel to $P_{0}$ and passing at a distance of $x(P)$ from it. Then, clearly, $S\left(C \cap P_{1}\right) \cdot 2 x(p)=\frac{4}{9} s\left(C \cap P_{0}\right) \cdot \frac{h(p)}{3}=\frac{4}{9} V(C)$.

Thus, the required planes are parallel to $P$ and pass at a distance of $x(P)$ from the center of symmetry of $K$.

The required continuous field of pairs of planes is constructed. By the same argument as in [5. Theorem 1], in this field we find a pair of symmetric planes $\left(P_{1}, P_{2}\right)$ with inscribed symmetric affine-regular octagons. By construction and by the estimate in Lemma 2, the convex hull of these octagons is the required affine-regular octagonal prism.

Corollaries. 1. Every c.s.c.b. $K \subset \mathbb{R}^{3}$ contains an affine image of a circular cylinder with the same center of symmetry and with volume at least $\frac{\pi}{9} V(K)$.

Indeed, such a cylinder is inscribed in the prism that exists by the theorem.

2. Every c.s.c.b. $K \subset \mathbb{R}^{3}$ contains a hexagonal prism of volume at least $\frac{2 \sqrt{2}}{9} V(K)$ and with centrally symmetric base.

Such a prism can be exhibited by taking the convex hull of the twelve vertices obtained by deleting four vertices of two symmetric lateral faces of the octagonal prism constructed in Theorem 3. 


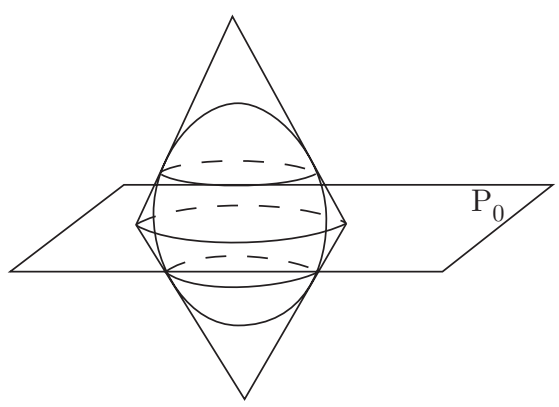

FiguRE 5 .

\section{$\S 6$. Approximation by DeCagonal and dodecagonal PRisms}

Theorem 4. Every c.s.c.b. $K \subset \mathbb{R}^{3}$ is contained in a circumscribed affine image of a regular decagonal prism that has volume at most $\left(3 / \cos 36^{\circ}\right) V(K)<3.7083 V(K)$ and is tangent to $K$ at the centers of the bases.

The proof follows from the fact that the orthogonal projection of $K$ to a plane is inscribed in an affine image of a regular decagon. In [5], it was proved that if in each plane $P \in G_{2}\left(\mathbb{R}^{3}\right)$ we have a centrally symmetric convex figure continuously depending on $P$, then an affine image of a regular decagon is inscribed in one of these figures. Applying this fact to the field of polar figures in the planes $P \in G_{2}\left(\mathbb{R}^{3}\right)$ (with respect to some continuous field of circles lying in these figures), we obtain the dual statement given above.

The volume estimate can be obtained by Lemma 1 if we take into account the fact that a convex figure of minimal area inscribed in a regular decagon is a regular pentagon the vertices of which are vertices of the decagon.

It is plausible that, about every c.s.c.b. $K \subset \mathbb{R}^{3}$, one can circumscribe an affine image of a regular dodecagonal prism that is tangent to $K$ at the centers of the bases (the volume of this prism is automatically less than or equal to $2 \sqrt{3} V(K)$ ). The author does not know how to prove this fact; however, the following statement is valid.

Theorem 5. Every c.s.c.b. $K \subset \mathbb{R}^{3}$ is contained in a circumscribed affine image of a dodecagonal right prism $C$ the base of which is a convex "semiregular" dodecagon with equal sides and with two alternating sizes of angles. This affine image is tangent to $K$ at the centers of the bases. The volume of the prism does not exceed $4 V(K)$.

From 8 it follows that, in each continuous field of c.s.c. figures in the planes belonging to $G_{2}\left(\mathbb{R}^{3}\right)$, there is a figure with an inscribed affine image of a semiregular dodecagon. The dual statement about a circumscribed affine image of a semiregular dodecagon is proved similarly.

The volume estimate is obtained by application of Lemma 1 and by taking into account the fact that a figure of minimal area inscribed in a semiregular dodecagon $K_{12}$ is the smallest of the two regular hexagons with vertices at vertices of the dodecagon, and its area does not exceed $\frac{3}{4} S\left(K_{12}\right)$.

Corollary. Any c.s.c.b. $K \subset \mathbb{R}^{3}$ is contained in a circumscribed affine image of a regular hexagonal prism that is tangent to $K$ at the centers of the bases and has volume at most $4 V(K)$. 
It is easy to check that these requirements are satisfied by the prism bounded by the bases and every other lateral face of the dodecagonal prism constructed in Theorem 5 .

Remark. If the above conjecture about the existence of a circumscribed affine-regular dodecagonal prism is valid, then, deleting every other lateral face, we obtain an affineregular hexagonal prism of volume at most $(2+\sqrt{3}) V(K)$ circumscribed about $K$.

\section{$\S 7$. Circumscribed direct hexagonal PRISms}

Theorem 6. Let $K \subset \mathbb{R}^{3}$ be a c.s.c.b. Then:

$1)$ there is a regular hexagonal prism circumscribed about $K$ and tangent to $K$ at points of great diagonals of the bases;

2) there is a direct hexagonal prism tangent to $K$ at the centers of the bases, circumscribed about $K$, and such that its base is a centrally symmetric hexagon with equal angles and a pair of adjacent sides of equal length.

It suffices to prove the theorem for strictly convex bodies $K$, which will be assumed in the sequel.

To prove the first part of the theorem, we consider the manifold $M$ of triples $\left(\bar{e}_{1}, \bar{e}_{2}, \bar{e}_{3}\right)$ of unit vectors with origin at $0 \in \mathbb{R}^{3}$ that lie in the same plane and form angles of $\frac{2 \pi}{3}$. It is obvious that $M \cong V_{2}\left(\mathbb{R}^{3}\right)$.

Now, we define a continuous map $F: V_{2}\left(\mathbb{R}^{3}\right) \rightarrow \mathbb{R}^{6}$ by putting $F\left(\bar{e}_{1}, \bar{e}_{2}, \bar{e}_{3}\right)=$ $\left(x_{1}, x_{2}, x_{3}, x_{4}, x_{5}, x_{6}\right)$, where $x_{1}, x_{2}$, and $x_{3}$ are the lengths of the orthogonal projections of $K$ to the vectors $\bar{e}_{1}, \bar{e}_{2}$, and $\bar{e}_{3}$, respectively. To define $x_{4}, x_{5}$, and $x_{6}$, we consider the unit vector $\bar{e}_{4}$ orthogonal to the vectors $\bar{e}_{1}, \bar{e}_{2}$, and $\bar{e}_{3}$ for which the triple $\left(\bar{e}_{1}, \bar{e}_{2}, \bar{e}_{4}\right)$ is right. Now, we define $x_{4}, x_{5}$, and $x_{6}$ as the coordinates of the orthogonal projections to $\bar{e}_{1}, \bar{e}_{2}$, and $\bar{e}_{3}$ of the point of $K$ the projection of which to $e_{4}$ is minimal (since $K$ is strictly convex, such a point is unique).

The cyclic group $\mathbb{Z}_{3}$ acts freely on the manifold $M$ by cyclic permutations of the vectors $\bar{e}_{1}, \bar{e}_{2}, \bar{e}_{3}$, and acts on $\mathbb{R}^{6}$ by simultaneous cyclic permutations in the first and second triples of coordinates of its points. By construction, the mapping $F$ preserves this action of $\mathbb{Z}_{3}$.

As was shown in 4, in this case we have $x_{1}=x_{2}=x_{3}$ and $x_{4}=x_{5}$ for some triple of vectors $\left(\bar{e}_{1}, \bar{e}_{2}, \bar{e}_{3}\right) \in M$ with $F\left(\bar{e}_{1}, \bar{e}_{2}, \bar{e}_{3}\right)=\left(x_{1}, x_{2}, x_{3}, x_{4}, x_{5}, x_{6}\right)$. Then the prism bounded by the eight support planes to $K$ that are orthogonal to the vectors $\bar{e}_{1}, \bar{e}_{2}, \bar{e}_{3}$, and $\bar{e}_{4}$, respectively, satisfies the conditions of statement 1 of the theorem.

The proof of the second part of the theorem is similar. The only difference is that, by [4], we have $x_{1}=x_{2}$ and $x_{4}=x_{5}=x_{6}$ for some triple of vectors $\left(\bar{e}_{1}, \bar{e}_{2}, \bar{e}_{3}\right) \in M$ with $F\left(\bar{e}_{1}, \bar{e}_{2}, \bar{e}_{3}\right)=\left(x_{1}, x_{2}, x_{3}, x_{4}, x_{5}, x_{6}\right)$.

Remark. Simple examples show that no estimates for the volume of the prism indicated in the theorem can be obtained in terms of the volume of $K$.

\section{REFERENCES}

[1] V. V. Makeev, Affine-inscribed and affine-circumscribed polygons and polyhedra, Zap. Nauchn. Sem. S.-Peterburg. Otdel. Mat. Inst. Steklov. (POMI) 231 (1995), 286-298; English transl., J. Math. Sci. (New York) 91 (1998), no. 6, 3518-3525. MR1434300 (98b:52004)

[2] A. Bielecki and K. Radziszewski, Sur les parallélépipèdes inscrits dans les corps convexes, Ann. Univ. Marie Curie-Skłodowska Sect. A 8 (1954), 97-100 (1956). MR.0081498 (18:412g)

[3] V. V. Makeev, Inscribed and circumscribed polyhedra of a convex body and related problems, Mat. Zametki 51 (1992), no. 5, 67-71; English transl., Math. Notes 51 (1992), no. 5-6, 469-472. MR.1186533 (93j:52013)

[4] - The Knaster problem and almost spherical sections, Mat. Sb. 180 (1989), no. 3, 424-431; English transl., Math. USSR-Sb. 66 (1990), no. 2, 431-438. MR0993234 (90d:55005) 
[5] - On geometric properties of three-dimensional convex bodies, Algebra i Analiz 14 (2002), no. 5, 96-109; English transl., St. Petersburg Math. J. 14 (2003), no. 5, 781-790. MR1970335 (2004c:52001)

[6] L. Fejes Tóth, Lagerungen in der Ebene, auf der Kugel und im Raum, Grundlehren Math. Wiss., vol. 65, Springer-Verlag, Berlin, 1953. MR0057566 (15:248b)

[7] B. Grunbaum, Affine-regular polygons inscribed in plane convex sets, Riveon Lematematika 13 (1959), 20-24. MR0108768 (21:7480)

[8] V. V. Makeev, Plane sections of convex bodies, and universal fibrations, Zap. Nauchn. Sem. S.Peterburg. Otdel. Mat. Inst. Steklov. (POMI) 280 (2001), 219-233; English transl., J. Math. Sci. (N.Y.) 119 (2004), no. 2, 249-256. MR1879268(2002k:52004)

Department of Mathematics and Mechanics, St. Petersburg State University, Universitetskil Prospekt 28, Stary ̌ Peterhof, St. Petersburg 198904, Russia

Received 5/MAY/2004

Translated by B. M. BEKKER 